\title{
Financial Decision Making in The Framework of Neuroscience / Anthropology with Review to The Pandemic and Climate Change
}

\author{
http://doi.org/10.21272/fmir.4(4).55-65.2020
}

\section{Ana Njegovanović}

Master of Economics, Lecturer at Faculty of Biotechnology in Zagreb; Faculty of Economics and Tourism, University of J. Dobrila in Pula, Croatia

\begin{abstract}
The purpose of this paper is interdisciplinary research of combinations of different disciplines of (natural) anthropology / neuroscience of consciousness and quantum physics and (social sciences) of financial decision making in the context of climate change and pandemics, which can be useful for finding new information, solving complex problems. The aim of this study is to provide insights into financial decision-making through the intertwining of anthropology / neuroscience and quantum physics in financial decision-making within COVID 19 and climate change and what their relationship / outcomes are. Human consciousness has slipped towards the collapse of convergent crises. Namely, health and climate change are intertwined. The causes of the COVID 19 crisis and climate change are common and their effects are approaching. The climatic situation and COVID-19, a zoonotic disease, are subject to human activity that has led to environmental degradation. Neither the climate crisis nor the zoonotic pandemic were unexpected. They have led to loss of life that could have been prevented by delayed, insufficient or wrong actions. Financial decision-making requires harmonizing public health improvements, creating a sustainable economic future and better protecting remaining natural resources and biodiversity Perhaps in this context financial simplification could be defined as coexistence of all options with different degrees of potential that we will choose them (it is a super position), other options cease to exist for us, when we enter the so-called zero of the desired option (the brain prepares our decisions). The results of the research showed us that COVID 19 and climate change have caused economic risks and uncertainties that have far-reaching and profound implications for financial decision-making as well as the financial services industry and its institutions. Extending tools through anthropology / neuroscience and quantum physics has given us knowledge of the need to connect both the natural and social sciences to understand the complex world around us.
\end{abstract}

Keywords: Anthropology, Neuroscience, Quantum physics, Financial Decision Making.

JEL Classification: I19, G41, D87, Q54.

This work is licensed under a Creative Commons Attribution 4.0 International License

Cite as: Njegovanović, A. (2020). Financial Decision Making in The Framework of Neuroscience / Anthropology with Review to The Pandemic and Climate Change. Financial Markets, Institutions and Risks, 4(4), 55-66. http://doi.org/10.21272/fmir.4(4).55-65.2020.

(C) The Author, 2020. This article is published with open access at Sumy State University.

\section{Introduction}

We explore combinations of different scientific disciplines (natural) anthropology / neuroscience of consciousness and quantum physics and (social sciences) financial decision making in the context of climate change and pandemics that can be useful for finding new information, solving complex problems.

Aims and purpose The research provides an overview of financial decision - making theory according to which decisions are made through various scientific disciplines in the context of climate change and pandemics, COVID 19.

Science is sometimes depicted as a methodical, painstaking search for truth, and good policymaking as the translation of those evidence-based truths into action. In pre-pandemic times, these assumptions sometimes (though not always) held. But the complexity of science and policymaking in the context of uncertainty has been brought into sharp focus by this pandemic (Lancaster K, Rhodes T, Rosengarten M. 2020).

Consciousness is entirely caused by neurobiological processes and is realized in brain structures. An essential feature of consciousness that we need to explain is unified qualitative subjectivity. Consciousness thus differs 
from other biological phenomena in that it has a subjective or first-person ontology, but this subjective ontology does not prevent us from having an epistemically objective science of consciousness. The two common approaches to consciousness are those that adopt the building block model, according to which each conscious field is composed of its different parts, and the unified field model, according to which we should try to explain the unique character of subjective states of consciousness.

To bridge the gap between the brain and consciousness, we need neural data, computational and psychological models, and philosophical analysis to identify the principles that link brain activity to brain activity with conscious experience. This article will focus on identifying such principles without shying away from neural details. The notion of neuroscientific explanation here is understood as providing informative answers to specific questions that can be answered by neuroscientific approaches. Accordingly, the theories and data to be considered will be organized around creating answers to two questions (Gazzaniga, Michael S. (2000).

Generic consciousness (Dennett, Daniel C., 2018): How can neural properties explain when a condition is conscious and not not?

Specific consciousness: How can neural properties explain the content of a conscious state?

In the naturalistic explanation of the phenomenon of consciousness, we face three main difficulties. The first stems from the fact that the explanation is not well defined. The second is the result of a still basic understanding of neural processes underlying higher cognitive functions, and the third is related to the "difficult problem" of consciousness research (Dennett, 2018), the intuition that even if we had a comprehensive view of neural correlates of consciousness (NCCP) we still would not could explain how firstperson experiences with the results of conscious processing, qualia, arise from neural interactions described from a third-person perspective.

The definition of consciousness is the totality of our awareness of bodily sensations, perceptions, emotions, thoughts and memories at a given moment. This is more considered a psychological definition of what consciousness is. However, the biological definition, which Gerald Edelman (Edelman, G. M. (2003) divided into what is called "primary consciousness" and "higher-order consciousness," shows the difference in degrees of consciousness.

Higher order consciousness differs from primary consciousness in that it involves actually recognizing an individual's own actions or preferences (we are aware that we are aware). Consciousness embodies the model of the personal and the past and the future, as well as the present; this is also known as "mental state awareness". In addition, higher-order consciousness exhibits direct awareness, noninferential, or immediate awareness of mental episodes without the involvement of sensory organs or receptors. According to Edelman (Edelman, 1987, 1993), it is believed that humans possess both primary consciousness and higher-order consciousness, and that the two coexist and merge interactions.

Consciousness is considered to be only a state of consciousness in a very general sense. However, the definition of what consciousness has received has been made by numerous contributions from many different fields of study. For example, psychology, psychiatry, neurophysiology, anthropology, behavioral science, and a new field called "cognitive science," which studies the nature of the various mental tasks and processes that enable them to be performed. It suneke variations on the growing definition of what consciousness is.

Another theory known as "neurological theory" or "Darwinian theory" holds that consciousness is the result of "progressive evolution" (centralization of the nervous system) possessed only by humans and higher mammals with this anatomical tendency.

"Biological theory" (Katz, B. F. 2013) is another definition that is more liberal and holds that all organisms essentially possess consciousness. A more extreme extension of biological theory is "cell theory," which believes that consciousness is a vital property of every cell. The final type of theory holds that consciousness is a fundamental property of all atoms; this is known as "atomistic theory". Philosophically, atomistic theory could imply that any DNA molecule (consisting of atoms) can be conscious in a way unknown to us and can actually do its own will. Thus, it is possible to have a "DNA awareness". Maybe this could have been the driving force behind evolution.

Understanding the neuroscience behind decision making can be helpful in targeting new behaviors and changing bad habits. Decision making is at the heart of our control. This implies the power to break patterns 
Financial Markets, Institutions and Risks, Volume 4, Issue 4, 2020 ISSN (online) - 2521-1242 ISSN (print) - 2521-1250

of behavior by simply making better decisions. We can change our opinion and our actions at any time. A February 2015 study by the Okinawa Institute of Science and Technology (OIST) of Japan University found that a key part of the brain involved in decision-making is the striatum, which operates hierarchically in its three different subregions.

The striatum is part of the basal ganglia, which form the inner core of the brain and process decision-making and further action. Neuroscientists divide the striatum into three regions: 1. Ventral (VS) 2. Dorsomedial (DMS) and 3. Dorsolateral (DLS). Each region plays a special role in: 1. motivation, 2. adaptable decisions, or 3. routine actions. We can briefly conclude that "Distinct neuronal representation in the dorsolateral, dorsomedial, and ventral part of the Striatum during fixed- and free-choice tasks". The prefrontal cortex shows activation throughout decision making.

Anthropology is the study of man, what man is, what defines him and his world, and what makes us special (or not). Anthropology is a hybrid discipline, relying on various methods borrowed from the natural, humanities, and social sciences. Our brains, our biological and cognitive characteristics allow us to create different cultural and social worlds. Our human nature is the nature of our brain, there is an intertwining of anthropology and neuroscience.

Anthropology explores human relationships with the environment, whether it be a physical or biological environment, a cultural-social environment, an early environment, or a linguistic environment. These different environments affect us, thus demonstrating two-way highway traffic between people and their surroundings. It remains unclear what allows such two-way traffic; this is the point at which anthropology approaches neuroscience, because the brain and its specific way of organizing its neural activity can enable and predispose such communication between humans and the environment.

Neuroscience has reached many disciplines in the humanities and social sciences. The integration of neuroscience and economics has created a new discipline of neuroeconomics, which models human decisionmaking processes in a financial context, and various other hybrid disciplines are prefixed with "Neuro", including "Neurolinguistics", "Neurotheology" and "Neuropsychoanalysis". Despite their different origins and contexts, all of these new disciplines share their interest in the brain as a potential origin and "place" of the phenomena they target.

Neuroscience reveals the necessary conditions for our ability to create different environments that anthropology deals with. Instead of reducing and incorporating anthropology into itself, neuroscience reaches its own limits where its empirical-experimental approach is complemented by other methods, such as social and conceptual anthropology. Therefore, instead of involving other fields, neuroscience can better pass by mapping its own boundaries and showing boundary stations, a knot between two disciplines.

What about the discipline of anthropology? Perhaps the empirical findings of neuroscience will be considered and used to better understand why the different environments we create take on a particular gestalt and not some others. Most importantly, given the brain and its purpose, including its specific neural code, anthropology can shed some light on the earth on which it stands. An encounter with neuroscience will allow anthropology to map its own boundaries, and thus where the two disciplines intersect.

To investigate such boundary cells, we need a special methodology, a transdisciplinary and thus truly hybrid method that allows us to connect different domains and contexts of neuroscience and anthropology. Here, too, anthropology provides fertile ground because, as mentioned at the outset, it itself relies on various methods derived from the natural, humanities, and social sciences. We need to systematically and non-reductionistically link these different methods to explain the intersection between man, the environment, and the brain, making it relevant to both anthropology and neuroscience.

We know that it is the brain itself that gives us information about ourselves as human beings and our different environment. We cannot avoid creating new environments, which has led to the development of a discipline with a methodology as diverse as anthropology. The connection between anthropology and neuroscience points to a deeper insight into the nature of man and his various environments. A transdisciplinary, and thus hybrid, methodology will promote and encourage interaction between neuroscience and anthropology by discovering common themes. This will allow us to supplement the anthropological order for self-search in the brain (Northoff, 2009) with the order for neuroscientific search of the brain in our different environments.

Financial decisions are among the most important life-shaping decisions that people make and which cognitive and neural processes affect them. Due to cognitive limitations and low average levels of financial literacy, 
many decisions violate sound financial principles. Thus households usually have an insufficiently diverse stock of stocks and low retirement savings rates. Investors are overexposed from past returns and trade too often. Even top corporate managers, who are usually highly educated, make decisions that are influenced by overconfidence and personal history. Many of these behaviors can be explained by known principles from cognitive science. Sudden collection of high-quality evidence - especially how practical it is.

We live in an unstable, insecure, complex, ambiguous and interconnected world - presenting both challenges and opportunities. For the first time in the World Economic Forum's annual report on global risks - which analyzes global, decade-long risks - the most likely global risks for 2020 were all environmental, including; extreme weather events, failure of climate action, natural disasters, great loss of biodiversity, and damage and disasters caused by human activity. With a little advance we can safely add global pandemics. The report identified a failure to act on climate mitigation and adaptation as a risk that is likely to have the greatest impact in the next 10 years.

Three important similarities between a global pandemic and chronic climate change: 1) both are exacerbated by human activity, 2) both can have a major impact both globally and locally, and 3) both require a global response.

The climate crisis and pandemic-COVID 19 (public health) face the greatest challenge of humanity. The catastrophic consequences of uncontrolled climate change are well known - from vast parts of the country becoming uninhabitable to crop failure and devastating forest fires and aspects of the current severe acute respiratory syndrome coronavirus-2 (SARS-CoV-2) and how different governments and institutions interpret and apply the same scientific This affects how local guidelines in each country are recorded and transmitted to health professionals and health professionals and to the general public.To use the language of music, the pandemic has brought us closer to ourselves.There is dissonance.Rhythms are random. We oppose opposite movements of rattle melodies. We seem to live in a labyrinth of smaller keys and open-type cadences. We move chromatically, step by step.A series of disagreements challenges us.Scientific knowledge and thus a solution is sought.

The COVID-19 pandemic sharpens the understanding of financial markets of the need to address threatening threats, including climate change. The rapid onset of the COVID-19 pandemic and the extent of the downtime globally indicate how suddenly the risks can crystallize. The existential threat posed by the situation with COVID-19 is clear. The threat posed by climate change is also real, but perhaps less tangible at this immediate time.

COVID-19 requires multidisciplinary teams. Organizations that have responded effectively have done so by quickly bringing together people from a range of disciplines, different skills and experiences, who understand all the details of how their organization works and what adjustments may be needed. Multidisciplinary teams are also vital to combating climate change and establishing a climate change risk management process.

This is likely to be the year when investors and financiers decide to incorporate climate transition analysis into their portfolios. Policymakers need to recognize that markets are moving much faster than they are at this critical level.

We have witnessed shocks in the oil and gas market in the last 70 years. By the end of July traditional oil and gas stocks in the S\&P 500 SPX, $+0.80 \%$ fell 45\%, Royal Dutch Shell RDS, $+3.58 \%$ reduced its dividend for the first time since World War II and BP BP, $+2,70 \%$ wrote off $\$ 17.5$ billion from the value of its assets. At the same time, clean energy stocks rose by just over $20 \%$, roughly the same as the technology sector (Project Syndicate September 25, 2020, the Worlds Opinion Page, Huw van Steenis- former Senior Adviser to the Governor of the Bank of England, Mark Carney, Chairman sustainable financing at UBS and a member of the Monetary and Financial System Council of the World Economic Forum).

Unprecedented economic transformation will be needed to achieve a prosperous, resilient and carbon-free economy and to maintain a secure and stable climate for humanity. Companies from each sector, as well as policy makers and financial institutions, have a role to play as policies, technologies and investments are together geared towards implementing socially inclusive transformation, pace and scale.

The scale of the climate challenge was recently highlighted by the UN Intergovernmental Panel on Climate Change (IPCC) Special Report on 1.5 Degrees, which cataloged the real environmental damage, human suffering and increased economic costs that would result if the average global temperature rises to exceed that 
level. The report made it clear that the world must reach 'net zero' emissions as soon as possible to stop contributing to climate change. The IPCC is an intergovernmental body through which, at the request of governments, thousands of experts review the latest sciences and analyzes to produce comprehensive assessments of what the academic opinion body indicates. IPCC reports clearly indicate the level of consensus and certainty around each finding.

\section{Methods}

The purpose of this section of the article is to comprehensively analyze the basic interdependence and interactions within financial decision making through the natural branches of science with the dynamics of the system generated by the virus and climate change.

Climate change and COVID-19 represent global development challenges and require responses in all countries (The Economist, 2020). Like COVID-19, climate change is an issue whose underlying causal processes and implications transcend boundaries, and the negative effects are felt far beyond the problem. Namely, the global North is a key part of the problem, and transitions in sustainability could benefit from multidirectional learning (Leach, 2015). COVID-19 stressed the need for action at the local and national levels, as well as multilateral cooperation to deliver global public goods. Global climate change management and COVID-19 is a challenge in an age of economic nationalism, but ensuring that efforts to adapt to the short and medium shocks of the COVID-19 pandemic are aligned with long-term climate change mitigation efforts require closer coordination among countries.

\section{Awareness / Quantum Physics and Quantum Information}

Human consciousness has been the nucleus of study in the fields of neuroscience, biology, psychology, physics, as well as philosophy. Scientists from all disciplines have tried to better understand the nature and meaning of consciousness and how our brain provides a sense of individual self. This goal has been approached in different ways in accordance with theoretical principles and research methods unique to each discipline. But despite these efforts, there is no widely accepted theory of how the brain facilitates self-awareness, intention, and abstract thought.

One of the more intriguing, but also controversial topics that R. Penrose dealt with, is certainly the one in which he tried to connect consciousness and quantum physics.

"He tried to link the collapse of wave functions to certain formations, microtubules in the human brain. This thesis has been much researched, but the brain system has been shown to be too large and too macroscopic for quantum mechanics to have any impact. Penrose covered the subject in a book. The Emperor's new mind," says our physicist.

Information is where abstract patterns meet the physical world. Because information is encoded into physical systems, their properties will crucially depend on the laws that govern those systems. Many aspects of defining our modern world - computers, satellites, telecommunications, smartphones, the Internet - currently use information that is encoded in classical degrees of freedom. However, physics is basically quantummechanical, so the final form of information is quantum information.

The goal of Quantum Information Science is to determine the laws of information in quantum mode: what properties information has and how those properties can be applied in a new and remarkable way. Quantum Internet, run on quantum information, will provide perfect encryption security and faster search algorithms. Quantum computers will be significantly more powerful than anything possible with a classical computer for key issues like chemistry simulation.

Quantum information science combines information theory and quantum mechanics, which has brought important technologies such as GPS and magnetic resonance imaging (MRI). It seeks to exploit unique quantum phenomena that have no classical counterpart, such as superposition and complexity. Potential advances in quantum information science and technology include new sensors, approaches to drug design and materials science, and processors that allow computing beyond the capabilities of classical machines. In addition, researchers are developing cryptography to protect information systems from quantum attacks.

The Copenhagen interpretation of quantum physics provides the foundations for the link between consciousness and quantum physics. Namely, the function of the quantum wave decays due to the conscious observer measuring the physical system. The thought experiment was initiated by Schroedinger's cat, as the level of absurdity of this way of thinking, except that it completely coincides with the evidence of what 
scientists observe on a quantum level. In summary, a fundamental platform that allows Schrödinger's cat to be both alive and dead, meaning that two particles can "talk to each other" even at a galaxy distance, might be able to explain human behavior.

Proponents of quantum consciousness point out that quantum uncertainty (a quantum system can never predict the outcome with certainty, but only as a probability from various possible states) would mean that quantum consciousness solves the problem or people do not actually have free will. So the argument goes, if human consciousness is governed by quantum-physical processes, then it is not deterministic and humans, therefore, have free will. Neuroscientist Sam Harris emphasizes, "If determinism is true, the future is set - and that includes all our future states of mind and our subsequent behavior. And to the extent that the law of cause and effect is subject to indeterminism - quantum or otherwise - we can't take any credit." for what is happening. There is no combination of these truths that seems compatible with the popular notion of free will.

Just as insecurity permeates the subatomic world, it is also present in our decision-making process. Unlike classical decision theories, the quantum world leaves room for a certain degree of uncertainty.

The view that "everything is relative" is quite closely related to the idea that "nothing in anthropology can be precisely defined". A closer look at current influence theory in

A closer look at the current influential in anthropology can distinguish many traces of quantum and relativistic logic. The latest "ontological turn" in sociocultural anthropology is largely based on relativism, which is pronounced perspectively and comparatively in this field (Alberti et al., 2011; Paleček and Risjord, 2012; Venkatesen, 2010; Viveiros de Castro, 2004)

Given the unprecedented urgency and new level of reflexivity introduced by climate change, anthropologists in research and practice must adopt a multi-scale, multinational and interdisciplinary approach. Anthropologists should deal with - the development of critical collaborative, multi-layered ethnography"climate ethnography".

Defining a catastrophe as a process / event that involves a combination of potentially destructive means from the natural and / or technological environment and the population in a socially and technologically produced state of vulnerability. From this basic understanding, three general thematic areas have developed: (a) approach to behavior and organizational response, (b) approach to social change, and (c) political economic / environmental approach, focusing on the historical-structural dimensions of vulnerability to vulnerability, especially in developing countries. The applied anthropological contribution in disaster management is discussed, as well as the research of danger perception and risk assessment. The article concludes with a discussion of the potentials in the study of danger and catastrophe for the construction of theory in anthropology, especially in matters of the relationship between man and the environment and sociocultural change.

Quantum information theory brings together ideas from classical information theory, quantum mechanics and informatics. The theorems and techniques of various branches of mathematics and mathematical physics, especially group theory, probability theory, and quantum statistical physics find application in this fascinating and fast-growing field.

Quantum information will help us in the accuracy of climate change calculations. Although quantum computing focuses on the growth of the number of qubits in a quantum computer, in reality there are many important factors in building the overall system that quantum solutions will accomplish. Hardware scaling, temperature control, software optimization, and many other considerations must be rethought in ways that allow large quantum computers to do the necessary, meaningful work of solving some of today's and tomorrow's biggest problems. Current and future problems are related to climate change. When a quantum computer is scaled to be able to deal with problems that classical computers cannot, what problems should we solve on it? Quantum researchers at Microsoft are creating concrete results, that is, how large quantum applications will accomplish tasks such as applications in chemistry, and they could help address one of the world's biggest challenges to date: climate change. Environmental sustainability that focuses on carbon, water, waste and biodiversity is one of the most ambitious carbon commitments set by any company.

With a human genome consisting of over 6.4 billion base pairs, quantum computing can prove to be an efficient way to process genetic data. Stephan Bekiranov, a computer biologist and associate professor, developed an algorithm that uses a quantum computer to study genetic diseases. This algorithm is designed to introduce 
efficiency into calculations by reducing the number of calculations performed in the operation. This discovery opens the possibility for researchers in the medical and genetic field to shred data faster and more efficiently, paving the way for new medical discoveries.

Unlike classical computing, where information is processed through bits - ones or zero - quantum computers analyze information through qubits, the basic units of quantum information.

For example if we have to process 2 bits, there are 4 possible states that can exist for it: $00,01,10$ and 11 . A classic computer would have to pass every possibility. However, using a quantum computer, all four states would be considered synchronously by applying the law of probability, with each state having an equal probability of occurrence.

This is the key to the efficiency of the Bekiran algorithm. Because genetic information is vast and often on a scale of billions of bits, the time required to process data by its algorithm decreases exponentially. "In biomedical research, we now have tons and tons of large data sets, and the ability to compute efficiently is where quantum computation fits into genetics," Bekirano.

\section{Corona Virus An Climate Change within Quantum Physics and The Financial Decision- Making Platform}

Scientists are trying to understand the virus and develop vaccines and treatments, Jon Cartwright looks at how physics plays an important role in that fight.

Physics-based techniques play a major role in the field of structural biology. The vast majority of the biological structures of macromolecules are obtained by X-ray crystallography. John Desmond Bernal and Dorothy Hodgkin (1934) recorded the first X-ray diffraction pattern of a crystallized protein, the digestive enzyme pepsin. Their work stemmed from the work of physicists like Wilhelm Röntgen, who discovered X-rays; Max von Laue, who discovered that the wavelengths of X-rays are comparable to interatomic distances and are therefore diffracted by crystals; and William Henry and William Lawrence Bragg, who showed how to use the diffraction pattern to analyze the corresponding crystal structure.

Individual biological molecules diffract X-rays, but very weakly. Crystallization, as Bernal and Hodgkin did for pepsin, is useful because it results in the repetition of a huge number of molecules in an ordered 3D grid, so that all their tiny signals amplify each other and become detected - photographic plates in the early days and active pixel detectors today. These signals are not images of molecules, because there are no materials that can be highly refracted and thus focus scattered X-rays. This is just a superficial insight into all the complexity of Covid 19, which requires knowledge of physics, biology, and therefore the natural sciences in order to formulate a platform for financial decision-making.

The COVID-19 pandemic requires an understanding of contemporary development challenges through a global rather than a narrower international development paradigm.

The global development paradigm rests on three main factors (Horner and Hulme, 2019; Horner, 2020). 1. The interconnectedness of modern capitalism means that the causal processes that shape 'development' have crossed the national and macro boundaries of the North and the South. 2. Climate change and sustainable development are key challenges facing the whole world. 3. Emerging patterns of global inequality over the last quarter of a century have crossed many North-South borders and created challenges common to all countries. Long-term issues are highlighted in the 21st century and are recognized in the Sustainable Development Goals (Fukuda-Parr and Muchhala, 2020; Gore, 2015) and the Paris Climate Agreement, which sets goals for all countries.

Research shows that sandstorms at high altitudes transmit harmful species of bacteria and fungi to thousands of miles of land and sea, also pointing to links to climate change.

Deserts are likely to become drier due to climate change over the next decade (Teruya Maki, an environmental microbiologist at Japan's Kanazawa University). Extreme sandstorms and wind patterns will encourage the dispersal of harmful colonies of bacteria and fungi. Fungi associated with dust events can increase existing allergen levels tenfold.

The scientific journal Geophysical Research: Atmospheres documents the microbial content of dust particles transported from the deserts of Central Asia to South Korea and Japan. In the process, researchers are showing 
the potential of dust to carry pathogens that can negatively affect both the natural ecosystem and human health, the American Geophysical Union (AGU) reports.

We know that microbes can survive and even thrive in the atmosphere. Microbes in the air have been reported to be present in an atmosphere with a density of up to a million cells per cubic meter (Frontiers in Bioengineering and Biotechnology, 2020). last.

Research shows that sandstorms at high altitudes transmit harmful species of bacteria and fungi to thousands of miles of land and sea, also pointing to links to climate change.

Deserts are likely to become drier due to climate change over the next decade (Teruya Maki, an environmental microbiologist at Japan's Kanazawa University). Extreme sandstorms and wind patterns will encourage the dispersal of harmful colonies of bacteria and fungi. Fungi associated with dust events can increase existing allergen levels tenfold.

The scientific journal Geophysical Research: Atmospheres documents the microbial content of dust particles transported from the deserts of Central Asia to South Korea and Japan. In The impact of the COVID 19 pandemic indicates that poverty reduction efforts in developing countries are being erased and rivalry is fostering and creating a chaotic world. Yet we have a chance to improve the situation if we align the future with creative capacities with the goal of building a safer, healthier, less divisive and cleaner future.

The process, researchers are showing the potential of dust to carry pathogens that can negatively affect both the natural ecosystem and human health, the American Geophysical Union (AGU) reports.

We know that microbes can survive and even thrive in the atmosphere. Microbes in the air have been reported to be present in an atmosphere with a density of up to a million cells per cubic meter (Frontiers in Bioengineering and Biotechnology, 2020).

The impact of the COVID 19 pandemic indicates that poverty reduction efforts in developing countries are being erased and rivalry is fostering and creating a chaotic world. Yet we have a chance to improve the situation if we align the future with creative cap On politics and macroeconomics, the impact of COVID 19 shows the announcement of fiscal stimulus ( $\$ 4$ trillion) as a way of financially responding to the immediate impact of a pandemic. There are also certain risks, such as distortions of state intervention, inefficiency, and moral hazards. Opportunities are also open to tackle inequality and improve public services, and to build more resilient, healthier and cleaner markets by tying aid to low-carbon commitments. Governments now have to make a historic choice: think ahead or encourage the next crisis. Recent research by IHS Markit shows that Covid-19 has strengthened existing policies and regulatory changes, with the European Union, the United Kingdom, Canada and China implementing measures during the pandemic in line with the less carbon-intensive pathway. Among the OECD countries, the exceptions are the United States and Mexico, which the US is lowering environmental standards, and Mexico is stopping new wind and solar projects. The EU is currently emerging as a leader. By the historic decision of the Presidency of the European Union "The Next Generation of the EU" on July 21, 2020 to give fiscal powers to Brussels, 750 billion euros - about one third of total spending - is earmarked for climate action. There are also indications that the European Central Bank under Christine Lagarde will better integrate climate risks into its activities, and several European countries have provided bold additional public funding for possible climate solutions, such as carbon or hydrogen capture. In the US, an investment plan for climate solutions in the amount of 2 trillion USD associated with clean energy and jobs.

\section{Discussion}

The aim of this paper is to gain insights into the complexity of financial decision-making through interdisciplinary research (involving natural and social sciences), intertwining climate change and the pandemic (COVID 19).

Interdisciplinary research is a combination of different disciplines such as a combination of natural and social sciences that can be useful for finding new information, solving complex problems (Nissani, 1997).

In natural and social sciences, researchers have different theoretical ideas and use different types of methodology, collect different types of data, ask different types of research questions, use different tools for data analysis and data validation and epistemological commitment. This will affect the results of interdisciplinary research, depending on the similarities or divergent outcomes in both disciplines. We must be aware that in the natural sciences the confirming hypothesis occurs autonomously from the information 
Financial Markets, Institutions and Risks, Volume 4, Issue 4, 2020 ISSN (online) - 2521-1242 ISSN (print) - 2521-1250

about that hypothesis, but in the social sciences the occurrence of a fact depends on the information of the hypothesis. Researchers from different disciplines can research the same topic, but when it is a complex topic, it is difficult to say which is superior to the other in all aspects.

Science indicates that current climate change is a threat to human lives, health and the economy. The decisions that governments now make have an impact on the strategic directions of companies and economies in the years to come. Pairing economic recovery with climate action at this extremely complex time will ensure that economies can recover stronger than before, while reducing emissions. Financial decisions as a framework to help companies also point to the need to integrate risk, establish science-based goals and accept the benefits of investing in technology as a basis for preserving the health of the national economy of people and nature.

The current environment is characterized by an increasingly complex set of pressures and demands from different stakeholder groups in the banking sector, increased expectations for social engagement and corporate citizenship, and radical uncertainty about the future. These factors complicate board decision-making and challenge the shareholder-led governance model that has led boards and business leaders for the past few decades.

Covid-19 complicated board decision-making and made it less subject to general rules and simple formulas. The "maximizing shareholder value" order simply doesn't have much to buy when it comes to deciding how much to invest in personal protective equipment to protect employee health or whether to turn a car production line into a fan production for the state in need. The pandemic has called into question many pre-crisis decisions made in the name of maximizing shareholder value, but which have left these companies tied to money, saddled with debt or otherwise ill-equipped to cope with the damage caused by COVID-19. In this new environment , committees increasingly have to rely on qualitative judgments in forming opinions and making decisions.

In summary, Covid 19 carries with it difficulties in making complex financial decisions. For example when paying dividends, making decisions would be relatively simple in the case of applying known dividend policies, following past practice, or selecting amounts based on shareholder expectations and company earnings in that period. But the situation with Covid-19, which has decimated the economy and uncertainty about the depth and duration of the crisis, the decision has become a complex issue of weighing and balancing multiple factors - at least for companies enough to take that into account at all. climate change joins the uncertain environment.

It is therefore necessary to start involving different scientific disciplines in order to understand the uncertainties and risks in order to find optimal outcomes with new methods (the aim of this paper).

\section{Conclusion}

This paper calls for a critical re-examination of a multitude of phenomena related to choice and decisionmaking, neuroscience, quantum physics. Reason and action can explain how choices are framed and constituted from the horizon of perceptions and expectations.

In interdisciplinary research, when scientists from the natural and social sciences come together to solve a problem, they could have a common interest within the problem, but they can define the same problem differently and approach it differently to have solutions that think about problem.

We have chosen an approach to the interconnectedness of natural and social sciences to provide insights into all the complexities of financial decision making through neuroscience / anthropology and quantum physics pointing to the need for knowledge in the coming times of technology and risk in solving complex problems.

Perhaps we should go into the analysis of financial decision-making with the words We are on the brink of a fundamental reshaping of finance. Larry Fink, President and CEO, BlackRock.

The financial system does not understand climate risk, physical risk or transition risk. Consistent data, standards, and methodologies are needed to improve this understanding, although testing for stress resistance and modeling scenarios will help move the understanding forward. From the COVID-19 situation can we learn how to better inform the financial system readily?

In financial services, there is awareness of the risks and opportunities associated with climate change, but often to a limited extent. It may be strongest in the insurance and wealth and asset management sectors, but even here the skills and experience associated with a full understanding of the risks associated with climate change are limited. Several organizations are able to set prices for climate change risk - presenting it in a financial 
perspective that allows organizations to anticipate future risks and supports complex asset allocation decisions. There are few financial services organizations that develop approaches to assessing the risk of carbon and granular climate sources to analyze the impact of temperature on portfolios.

Whether we are fighting a pandemic or a climate crisis, we need scientific solutions through the inclusion of natural and social branches of science as the key to new information, a solution to a problem. Namely, there is knowledge that 2020 will be a turning point for the climate change crisis, which will require a radical reversal of the current trajectory in global greenhouse gas emissions. However, this year we are also facing a global health crisis. The decisions that are being made now will affect us but generations to come, including our ability to stop global warming. There is no established link between COVID 19 and climate change. But the way we change the planet will make the spread of some new diseases likely. The coronavirus pandemic is a tragedy and its consequences will be felt for a long time. Yet, although global health conditions will eventually return to normal, our environment will never do so.

The COVID-19 pandemic affected all aspects of society. Financial services are no exception, as companies face issues of prudential and operational resilience and seek to protect vulnerable customers. This crisis has heightened the importance of ESG (environmental, social and governance) risks facing the sector, with deep implications for the investment, operational and balance sheet risk profile.

\section{References}

1. Andersen, R. A., Kristen, N. A., Hwang, E. J., Hauschild, M. (2014). Optic Ataxia: From Balint's Syndrome to the Parietal Reach Region, Neuron, 81(5), 967-83. doi:10.1016/j.neuron.2014.02.025. Retrieved from: www.vis.caltech.edu/documents/9178/andersen2014.pdf

2. Behrmann, M. Plaut, D. C. (2013). Distributed Circuits, Not Circumscribed Centers, Mediate Visual Recognition. Trends in Cognitive Sciences, 17(5): 210-219. doi:10.1016/j.tics.2013.03.007. Retrieved from: www.ncbi.nlm.nih.gov/pubmed/23608364

3. Dennett, D. C. (2018). Facing up to the Hard Question of Consciousness. Philosophical Transactions of the Royal Society B: Biological Sciences, 373 (1755): 20170342. doi: 10.1098 / rstb.2017.0342. Retrieved from: www.ncbi.nlm.nih.gov/pubmed/23608364

4. Edelman, G. M., and Tononi, G. (2000). A Universe of Consciousness. New York: Basic Books. Retrieved from: www.scirp.org/reference/referencespapers.aspx?

5. Edelman, G. M. (2003). Naturalizing consciousness: a theoretical framework. Proc. Nat. Acad. Sci. U.S.A. 100, 5520-5524. Retrieved from: www.researchgate.net/publication/10797704.

6. Edelman, G. M. (1993). Neural Darwinism: selection and reentrant signaling in higher brain function. Neuron 10, 115-125. Retrieved from: www.semanticscholar.org/paper/Neural-Darwinism

7. Fetsch, Christopher R., Roozbeh Kiani, William T. Newsome, and Michael N. Shadlen, 2014, "Effects of Cortical Microstimulation on Confidence in a Perceptual Decision", Neuron, 83(4): 797-804. doi:10.1016/j.neuron.2014.07.011. Retrieved from: www.cns.nyu.edu/.../Fetsch_Kiani Newsome Shadlen_2014.pdf

8. Fukuda- Parr, Muchalla B. (2020) The Southern origins of sustainable development goals: Ideas, actors, aspirations, World Development, 126. Article 104706. Retrieved from: www.researchgate.net/ publication $/ 337145120$

9. Gazzaniga, Michael S. (2000). Cerebral specialization and interhemispheric communication: Does the corpus callosum enable the human condition? Brain, 123 (7) 1293-1326. Retrieved from: https://academic.oup.com/brain/article/123/7/1293/380106

10. Gore. C. (2015). Towards Sustainable Development Goals and a new global development paradigm, Journal of International Development, 27(6), 717-732. Retrieved from: https://onlinelibrary.wiley.com/ doi/ abs/10.1002/jid.3109

11. Histed, M. H., Amy, M. N., Maunsell, H. R. (2013). Insights into Cortical Mechanisms of Behavior from Microstimulation Experiments, Progress in Neurobiology, Special Issue: Conversion of Sensory Signals into Perceptions, Memories and Decisions, 103(April): 115-130. doi:10.1016/j.pneurobio.2012.01.006. Retrieved from: https://philpapers.org/sep/consciousness-neuroscience/?refresh=1

12. Horner, R. Hulme, D. (2019). From International to global development: New geographies of 21st century development, Development and Change, 50(2), 347-378. Retrieved from: https://onlinelibrary.wiley.com/doi/abs/10.1111/dech.12379 
13. Horner, R. (2020). Towards a new paradigm of global development? Beyond the limits of international development, Progress in HumanGeography, 44(3), 415-436. Retrieved from: https://journals.sagepub.com/doi/full/10.1177/0309132519836158

14. Katz, B. F. (2013). An embarrassment of theories. J. Conscious. Stud. 20, 43-69. Retrieved from: www.frontiersin.org/articles/10.3389/fpsyg.2013

15. Kozuch, B. (2014). Prefrontal Lesion Evidence against Higher-Order Theories of Consciousness", Philosophical Studies, 167(3), 721-746. doi:10.1007/s11098-013-0123-9. Retrieved from: https://link.springer.com/article/10.1007/s11098-013-0123-9

16. Lancaster, K., Rhodes T., Rosengarten, M. (2020). Making evidence and policy in public health emergencies: lessons from COVID-19 for adaptive evidence-making and intervention. Evidence \& Policy: A Journal of Research, Debate and Practice. Retrieved from: www.bmj.com/content/370/bmj.m3349

17. Leopold, D. A. (2012). Primary Visual Cortex: Awareness and Blindsight, Annual Review of Neuroscience, 35, 91-109. doi:10.1146/annurev-neuro-062111-150356

18. Maniscalco, B. Hakwan, L. (2012). A Signal Detection Theoretic Approach for Estimating Metacognitive Sensitivity from Confidence Ratings, Consciousness and Cognition, 21(1), 422-430. doi:10.1016/j.concog.2011.09.021 Retrieved from: www.sciencedirect.com/science/article/pii/S.

19. Odegaard, B., Knight, R. T., Hakwan, L. (2017). Should a Few Null Findings Falsify Prefrontal Theories of Conscious Perception? Journal of Neuroscience, 37(40): 9593-9602. doi:10.1523/JNEUROSCI.321716.2017

20. Shea, N. (2014). Neural Signalling of Probabilistic Vectors, Philosophy of Science, 81(5), 902-913. doi:10.1086/678354. Retrieved from: https://www.morganstanley.com/im/ publication/insights/ investment-insights/ii_weatheringthestorm_us.pdf. 